\title{
Hyper-X Mach 10 Trajectory Reconstruction
}

\author{
Christopher D. Karlgaard* \\ Analytical Mechanics Associates, Inc., Hampton, VA 23666 \\ John G. Martin ${ }^{\dagger}$ and Paul V. Tartabini ${ }^{\ddagger}$ \\ NASA Langley Research Center, Hampton, VA 23681 \\ Mark N. Thornblom ${ }^{\S}$ \\ Analytical Mechanics Associates, Inc., Hampton, VA 23666
}

\begin{abstract}
This paper discusses the formulation and development of a trajectory reconstruction tool for the NASA $\mathrm{X}-43 \mathrm{~A} / \mathrm{Hyper}-\mathrm{X}$ high speed research vehicle, and its implementation for the reconstruction and analysis of flight test data. Extended Kalman filtering techniques are employed to reconstruct the trajectory of the vehicle, based upon numerical integration of inertial measurement data along with redundant measurements of the vehicle state. The equations of motion are formulated in order to include the effects of several systematic error sources, whose values may also be estimated by the filtering routines. Additionally, smoothing algorithms have been implemented in which the final value of the state (or an augmented state that includes other systematic error parameters to be estimated) and covariance are propagated back to the initial time to generate the best-estimated trajectory, based upon all available data. The methods are applied to the problem of reconstructing the trajectory of the Hyper-X vehicle from data obtained during the Mach 10 test flight, which occurred on November 16th 2004.
\end{abstract}

\section{Introduction}

$\prod$ NASA Hyper-X Program is a joint Langley Research Center and Dryden Flight Research Center 1 effort to develop a hypersonic airbreathing research vehicle, powered by an airframe-integrated supersonic combustion ramjet (scramjet) propulsion system. Major objectives of the program are to acquire data on the operation of the scramjet propulsion system and to demonstrate controlled hypersonic flight technologies. A secondary goal is to evaluate the experimental and computational techniques used in the design and analysis of the Hyper-X vehicle in order to facilitate future hypersonic and launch vehicle programs. ${ }^{1,2}$

The flight experiment mission profile is shown in Fig. 1, and takes place in the Western Aeronautical Test Range (WATR) over the Pacific Ocean. The experiment begins with a boost phase, where the HyperX Launch Vehicle (HXLV) stack (shown in Fig. 2), consisting of the X-43A/Hyper-X Research Vehicle (HXRV), shown in Fig. 3 and described in Ref. 3, mated to a modified Pegasus booster as described in Ref. 4, is dropped from the NASA B-52 aircraft at an altitude of approximately 40,000 ft and ascends to a high altitude. At this point, the HXRV separates from the stack, stabilizes, and the scramjet propulsion experiment begins, lasting several seconds. Following the engine test, the vehicle follows a descent trajectory that includes various maneuvers that are optimized for the post-flight extraction of stability and control derivatives and other aerodynamic properties. Three flights were planned, two at a test condition of Mach 7 and one at Mach 10. The first flight on June 2, 2001, ended with a failure during the boost phase of the trajectory. The second and third tests, on March 27 and November 16, 2004, were both successful. Further information on these test flights and preliminary results may be found in Refs. 5-7.

A critical component of the post-flight data reduction and analysis tasks to determine propulsion system performance and the extraction of various aerodynamic coefficients is the reconstruction of the vehicle

\footnotetext{
*Project Engineer, Senior Member AIAA.

$\dagger$ Aerospace Engineer, Exploration Concepts Branch.

¥Aerospace Engineer, Exploration Concepts Branch.

$\S$ Project Engineer.
} 


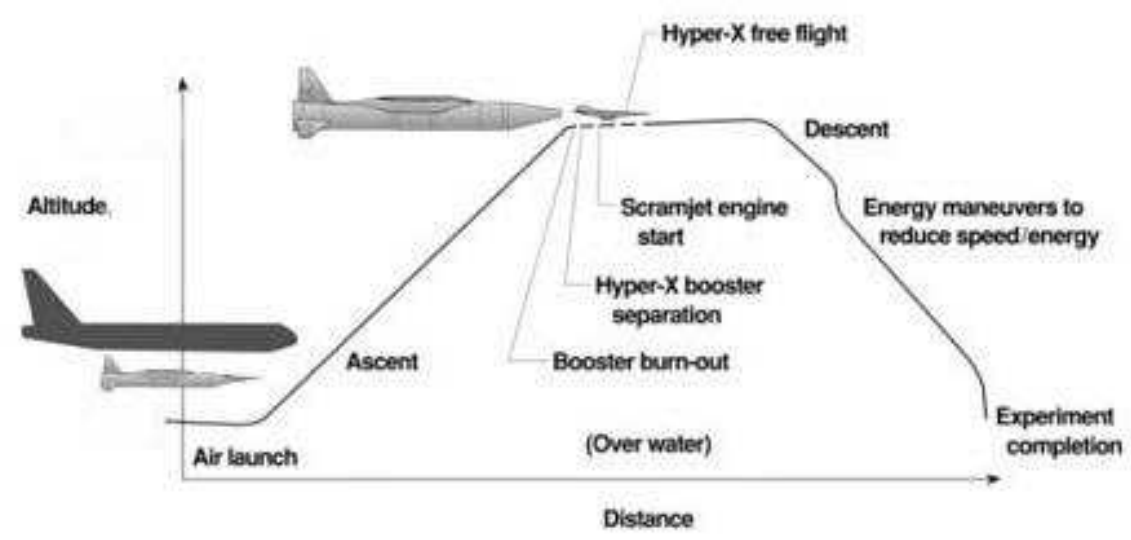

Figure 1. Hyper-X Flight Trajectory

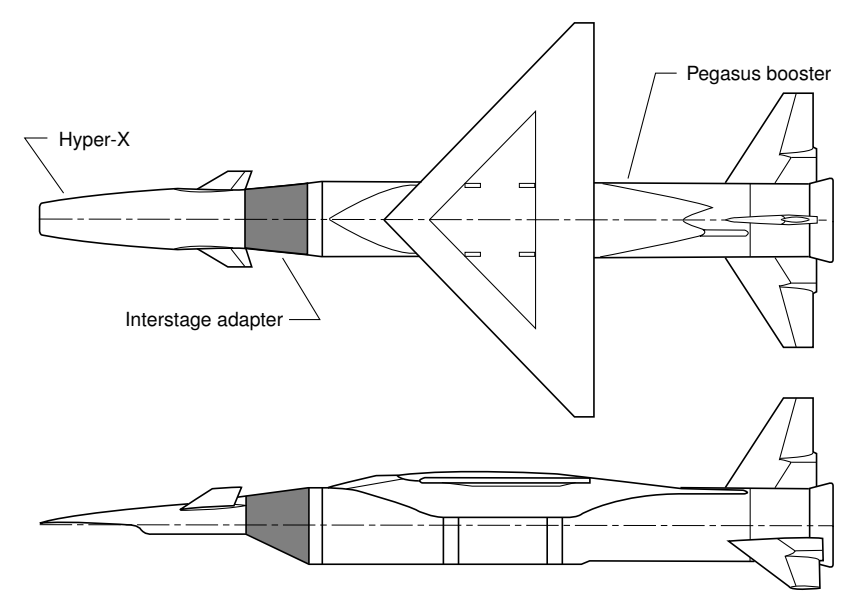

Figure 2. Hyper-X Launch Vehicle Stack Configuration

trajectory and associated atmospheric flight conditions. The trajectory reconstruction process uses in-flight telemetry data combined with a variety of redundant measurements to generate maximum-likelihood estimates of the vehicle position, velocity, and orientation time histories, along with estimates of the uncertainty associated with them. The Hyper-X vehicle has many instruments that gather data to be used for this process, such as the Inertial Measurement Unit (IMU), Global Positioning System (GPS), and ground-based radar tracking data.

A tool has been developed for the purpose of reconstructing the trajectory of the Hyper-X vehicle based on multiple data sources. The reconstruction approach is a modernization of that given in Refs. 11,12 and uses a recursive, minimum variance filtering algorithm to process accelerometer and gyroscope measurements recorded by the IMU during the flight, as well as position and velocity measurements from outside sources, such as ground based radar tracking stations, to determine a maximum-likelihood trajectory and user defined systematic error sources along with an estimate of the accuracy of these parameters. The data are processed through the use of an Extended Kalman Filter $(\mathrm{EKF})^{13}$ for a forward and backward pass through the data. The results of the two filtering passes are combined using the Fraser-Potter smoothing method ${ }^{13,14}$ to determine the final trajectory estimate as a function of time. The method also allows the user to specify which systematic error sources should be estimated, such as an accelerometer bias, and is general with the type of outside tracking measurements available.

The details of the trajectory reconstruction code and its application to the Mach 7 flight of the Hyper-X vehicle have been described in Refs. 8-10. This paper discusses the application of the tool to the data acquired during the Mach 10 test flight. A brief review of the reconstruction method is provided first. 


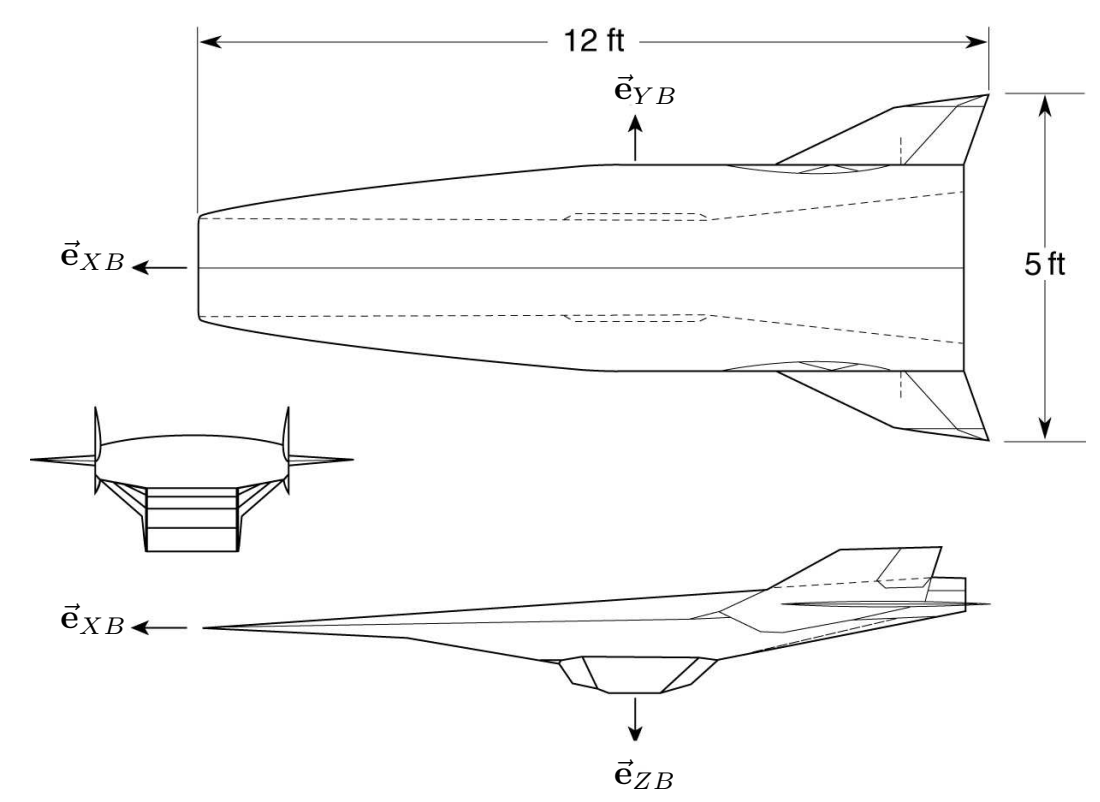

Figure 3. Hyper-X Research Vehicle Configuration

\section{Reconstruction Method}

The reconstruction method is based on the Extended Kalman filter/smoother, which is essentially a nonlinear recursive least-squares algorithm. The method makes use of a forward filter and backward filter to process data acquired during the flight. The forward filter is a predictor-corrector approach in which the trajectory predictions are formed by direct numerical integration of the onboard accelerometer and gyroscope measurements, and trajectory corrections are provided by the processing of Global Positioning System position and velocity measurements acquired by the onboard receiver. Smoothing algorithms have been implemented in which the final estimate of the trajectory and its associated covariance are propagated back to the initial time to generate the best-estimated trajectory, based upon all available data. The estimation process also develops estimates of various IMU error parameters, such as bias and scale factor errors. The final product of this process is a planet-relative trajectory along with all uncertainties. The specifics of the filtering and smoothing equations are discussed as follows.

\section{A. Filtering and Smoothing Equations}

The current section shall be concerned with the problem of estimating the state of a nonlinear system of ordinary differential equations

$$
\dot{\mathbf{x}}(t)=\mathbf{f}(\mathbf{x}(t), \mathbf{v}(t), t)
$$

where $\mathbf{x}$ is the matrix of the system states, $\mathbf{f}(\mathbf{x}, \mathbf{v}, t)$ is a nonlinear function that describes the evolution of the system state, and $\mathbf{v}$ is a random process with covariance matrix $\mathbf{Q}$ given by $\mathbf{Q}=E\left(\mathbf{v} \mathbf{v}^{T}\right)$ and mean $\overline{\mathbf{v}}=E(\mathbf{v})$, where $E$ is the expectation of the random variable. The state estimation procedure requires knowledge of independent observations of some quantity that may be related to the value of the state by the nonlinear algebraic equations

$$
\mathbf{y}(t)=\mathbf{g}(\mathbf{x}(t), \mathbf{w}(t), t)
$$

given at discrete time intervals, where $\mathbf{y}$ is the value of the observation, $\mathbf{g}$ is the nonlinear relationship between the states and the observations, and $\mathbf{w}$ is a random measurement noise variable with covariance matrix $\mathbf{R}$ and mean $\overline{\mathbf{w}}$.

The extended Kalman filter (EKF) is a somewhat ad-hoc modification of the linear Kalman filter in which the linear state transition is replaced by a numerical integration of the full nonlinear dynamic model in between measurement updates, before applying the Kalman update at each measurement time. In addition, the nonlinear relationship between the state and measurement data is used to calculate the residuals rather than the linear approximate relationship. The EKF uses the current estimated state as the reference conditions for computing the system dynamics matrices required to calculate the Kalman gain matrix at 
each time step. The algorithm is given by ${ }^{13}$

$$
\hat{\mathbf{x}}_{k}=\overline{\mathbf{x}}_{k}+\mathbf{K}_{k}\left[\mathbf{y}_{k}-\mathbf{g}\left(\overline{\mathbf{x}}_{k}, \overline{\mathbf{w}}_{k}, t_{k}\right)\right]
$$

where $\overline{\mathbf{x}}_{k}$ is the predicted value of the state at time increment $k, \mathbf{y}_{k}$ is the value of the measurement data, and $\mathbf{g}\left(\overline{\mathbf{x}}_{k}, \overline{\mathbf{w}}_{k}, t_{k}\right)$ is the predicted value of the measurement data at time $t_{k}$, following from the propagation of the state estimate from time $t_{k-1}$ to time $t_{k}$. The gain and covariance equations are given by

$$
\begin{aligned}
\mathbf{K}_{k} & =\overline{\mathbf{P}}_{k} \mathbf{C}_{k}^{T}\left(\mathbf{C}_{k} \overline{\mathbf{P}}_{k} \mathbf{C}_{k}^{T}+\mathbf{D}_{k} \mathbf{R}_{k} \mathbf{D}_{k}^{T}\right)^{-1} \\
\hat{\mathbf{P}}_{k} & =\left(\mathbf{I}-\mathbf{K}_{k} \mathbf{C}_{k}\right) \overline{\mathbf{P}}_{k}
\end{aligned}
$$

where $\overline{\mathbf{P}}_{k}$ is the solution of the matrix differential equation

$$
\begin{aligned}
\dot{\overline{\mathbf{P}}}(t) & =\mathbf{A} \overline{\mathbf{P}}+\overline{\mathbf{P}}^{T} \mathbf{A}^{T}+\mathbf{B} \mathbf{Q B} \mathbf{B}^{T} \\
\overline{\mathbf{P}}\left(t_{k-1}\right) & =\hat{\mathbf{P}}_{k-1}
\end{aligned}
$$

at time $t_{k}$, calculated through numerical integration. The matrices $\mathbf{A}, \mathbf{B}, \mathbf{C}$ and $\mathbf{D}$ used in the Kalman gain calculations are found by linearizing the system dynamics about the current state estimate, $\hat{\mathbf{x}}_{k}$, so that

$$
\begin{aligned}
\mathbf{A}(t) & =\left.\frac{\partial \mathbf{f}}{\partial \mathbf{x}}\right|_{\mathbf{x}=\hat{\mathbf{x}}_{k}, \mathbf{v}=\mathbf{0}, t} \\
\mathbf{B}(t) & =\left.\frac{\partial \mathbf{f}}{\partial \mathbf{v}}\right|_{\mathbf{x}=\hat{\mathbf{x}}_{k}, \mathbf{v}=\mathbf{0}, t} \\
\mathbf{C}(t) & =\left.\frac{\partial \mathbf{g}}{\partial \mathbf{x}}\right|_{\mathbf{x}=\hat{\mathbf{x}}_{k}, \mathbf{w}=\mathbf{0}, t} \\
\mathbf{D}(t) & =\left.\frac{\partial \mathbf{g}}{\partial \mathbf{w}}\right|_{\mathbf{x}=\hat{\mathbf{x}}_{k}, \mathbf{w}=\mathbf{0}, t}
\end{aligned}
$$

Note that in the formulation of the filtering equations, the state $\mathbf{x}$ could include both dynamic states as well as constant model parameters to be estimated. Likewise, the input noise $\mathbf{v}$ could include random inputs to reflect the model uncertainty (process noise), or the uncertainty associated with model parameters that are not included in the state $\mathbf{x}$. The uncertain parameters from the latter case are commonly known as "consider parameters." The two noise inputs to the system dynamics both seek to degrade the state covariance matrix in order to reflect uncertainty in the model, but are different in the sense that the process noise accounts for effects that are unknown whereas the consider parameters account for effects that are known but uncertain. A Kalman filter designed to include parametric uncertainty is typically known as a Kalman-Schmidt filter.

The EKF algorithm implemented in this paper may be used for both a forward pass and a backward pass through the measurement data. The final estimate from the forward pass through the data is used as the initial condition for the backward pass. The final trajectory is then computed as a linear combination of the forward and backward passes using the Fraser-Potter method. ${ }^{13,14}$ The optimal smoothed solution at a time increment $k$ is given by

$$
\begin{aligned}
\hat{\mathbf{P}}_{k} & =\left[\hat{\mathbf{P}}_{f_{k}}^{-1}+\hat{\mathbf{P}}_{b_{k}}^{-1}\right]^{-1} \\
\hat{\mathbf{x}}_{k} & =\hat{\mathbf{P}}_{k}\left[\hat{\mathbf{P}}_{f_{k}}^{-1} \hat{\mathbf{x}}_{f_{k}}+\hat{\mathbf{P}}_{b_{k}}^{-1} \hat{\mathbf{x}}_{b_{k}}\right]
\end{aligned}
$$

where $\hat{\mathbf{P}}$ is the smoothed covariance estimate, and $\hat{\mathbf{x}}$ is the smoothed state estimate, $\hat{\mathbf{P}}_{f}$ and $\hat{\mathbf{P}}_{b}$ are the covariance estimates from the forward and backward filter, respectively, and $\hat{\mathbf{x}}_{f}$ and $\hat{\mathbf{x}}_{b}$ are the state estimates from the forward and backward filter. By inspecting Eqs. (12-13) it is apparent that the smoother combines the forward estimate at time $t_{k}$, which makes use of all measurement data from the beginning of the data set to time $t_{k}$, with the backward estimate at time $t_{k}$, which makes use of all data from time $t_{k}$ to the end of the interval. In other words, the smoother makes use of all available data at each point of interest.

\section{B. Dynamic Model}

This section discusses the dynamic model that is used for numerically propagating the state estimates between the Kalman filter updates. The equations of motion are based upon Newton's laws of motion, 
and are developed in a local, non-inertial coordinate system. The inertial acceleration and angular rate measurements recorded during the flight are used in place of detailed aerodynamic and propulsion force and moment models. An approximate representation of the complete gravitational potential is used to develop an expression for the gravitational force acting upon the vehicle. The following summary begins with the definition of various coordinate systems.

\section{Coordinate Systems}

This section provides a brief review of the coordinate systems used to develop the nonlinear equations of motion used in the trajectory reconstruction process. Firstly, the Earth Centered Inertial (ECI) frame is the frame fixed at the center of the Earth, or any other primary body of interest. The fundamental plane of this coordinate system is the equatorial plane of the Earth, with the $x$-axis, $\overrightarrow{\mathbf{e}}_{X I}$, aligned with the vernal equinox, the $z$-axis, $\overrightarrow{\mathbf{e}}_{Z I}$, in the direction of the planetary axis of rotation, and $y$-axis, $\overrightarrow{\mathbf{e}}_{Y I}$, chosen in such a way as to complete a right-handed coordinate system. This frame is obviously not truly inertial, although for short periods of time the inertial assumption is a valid and common approximation (see Ref. 15).

The Earth Centered Earth Fixed $(E C E F)$ frame is the coordinate system whose origin is located at the center of the Earth, similar to the ECI frame, but the coordinate axes are fixed with respect to the planet surface. The $x$-axis, $\overrightarrow{\mathbf{e}}_{X F}$, and the $z$-axis, $\overrightarrow{\mathbf{e}}_{Z F}$, are aligned with the prime meridian and the axis of planetary rotation, respectively. This definition of the ECEF frame is consistent with the World Geodetic System of 1984 (WGS-84). The ECEF and ECI frames may be related by the simple transformation

$$
\begin{array}{r}
\left\{\begin{array}{c}
\overrightarrow{\mathbf{e}}_{X F} \\
\overrightarrow{\mathbf{e}}_{Y F} \\
\overrightarrow{\mathbf{e}}_{Z F}
\end{array}\right\}=\mathbf{G}_{E C I 2 E C E F}\left\{\begin{array}{c}
\overrightarrow{\mathbf{e}}_{X I} \\
\overrightarrow{\mathbf{e}}_{Y I} \\
\overrightarrow{\mathbf{e}}_{Z I}
\end{array}\right\} \\
\mathbf{G}_{E C I 2 E C E F}=\left[\begin{array}{ccc}
\cos \lambda & \sin \lambda & 0 \\
-\sin \lambda & \cos \lambda & 0 \\
0 & 0 & 1
\end{array}\right]
\end{array}
$$

The geocentric $(G C)$ frame is one whose origin is located at the center of mass of the vehicle, and whose $z$-axis, $\overrightarrow{\mathbf{e}}_{Z C}$, is aligned in the direction of the origin of the $E C I$ (and $E C E F$ ) frame. The $x$-axis of the this frame, $\overrightarrow{\mathbf{e}}_{X C}$, is perpendicular to the $z$-axis and in the plane defined by $\overrightarrow{\mathbf{e}}_{Z F}$ and $\overrightarrow{\mathbf{e}}_{Z C}$, and oriented such that the scalar product of $\overrightarrow{\mathbf{e}}_{X C}$ and $\overrightarrow{\mathbf{e}}_{Z F}$ is positive. The $G C$ frame may be related to the $E C E F$ frame by the transformation

$$
\begin{aligned}
\left\{\begin{array}{c}
\overrightarrow{\mathbf{e}}_{X C} \\
\overrightarrow{\mathbf{e}}_{Y C} \\
\overrightarrow{\mathbf{e}}_{Z C}
\end{array}\right\} & =\mathbf{G}_{E C E F 2 G C}\left\{\begin{array}{l}
\overrightarrow{\mathbf{e}}_{X F} \\
\overrightarrow{\mathbf{e}}_{Y F} \\
\overrightarrow{\mathbf{e}}_{Z F}
\end{array}\right\} \\
\mathbf{G}_{E C E F 2 G C} & =\left[\begin{array}{ccc}
-\sin \phi & 0 & \cos \phi \\
0 & 1 & 0 \\
-\cos \phi & 0 & -\sin \phi
\end{array}\right] \cdot\left[\begin{array}{ccc}
\cos \theta & \sin \theta & 0 \\
-\sin \theta & \cos \theta & 0 \\
0 & 0 & 1
\end{array}\right]
\end{aligned}
$$

The geocentric frame is sometimes called the topocentric or local-vertical frame. The $E C I, E C E F$, and $G C$ frames are shown in Fig. 4.

A frame similar to the $G C$ frame is the geodetic $(G D)$ frame, sometimes known as the topodetic, localhorizontal, or North-Earth-Down $(N E D)$ frame. This frame is one in which the origin is located at the center of mass of the vehicle, and the $x$-axis, $\overrightarrow{\mathbf{e}}_{X D}$, points in the direction tangent to the surface of the Earth, in the direction of the North pole. The $z$-axis, $\overrightarrow{\mathbf{e}}_{Z D}$, is aligned perpendicular to the $x$-axis and oriented downward and is perpendicular to the line tangent to the surface of the planet, while the $y$-axis, $\overrightarrow{\mathbf{e}}_{Y D}$ is shared with the $G C$ frame. The $G D$ frame may be related to the $G C$ frame by the transformation

$$
\begin{aligned}
\left\{\begin{array}{l}
\overrightarrow{\mathbf{e}}_{X D} \\
\overrightarrow{\mathbf{e}}_{Y D} \\
\overrightarrow{\mathbf{e}}_{Z D}
\end{array}\right\} & =\mathbf{G}_{G C 2 G D}\left\{\begin{array}{l}
\overrightarrow{\mathbf{e}}_{X C} \\
\overrightarrow{\mathbf{e}}_{Y C} \\
\overrightarrow{\mathbf{e}}_{Z C}
\end{array}\right\} \\
\mathbf{G}_{G C 2 G D} & =\left[\begin{array}{ccc}
\cos \epsilon & 0 & \sin \epsilon \\
0 & 1 & 0 \\
-\sin \epsilon & 0 & \cos \epsilon
\end{array}\right]
\end{aligned}
$$




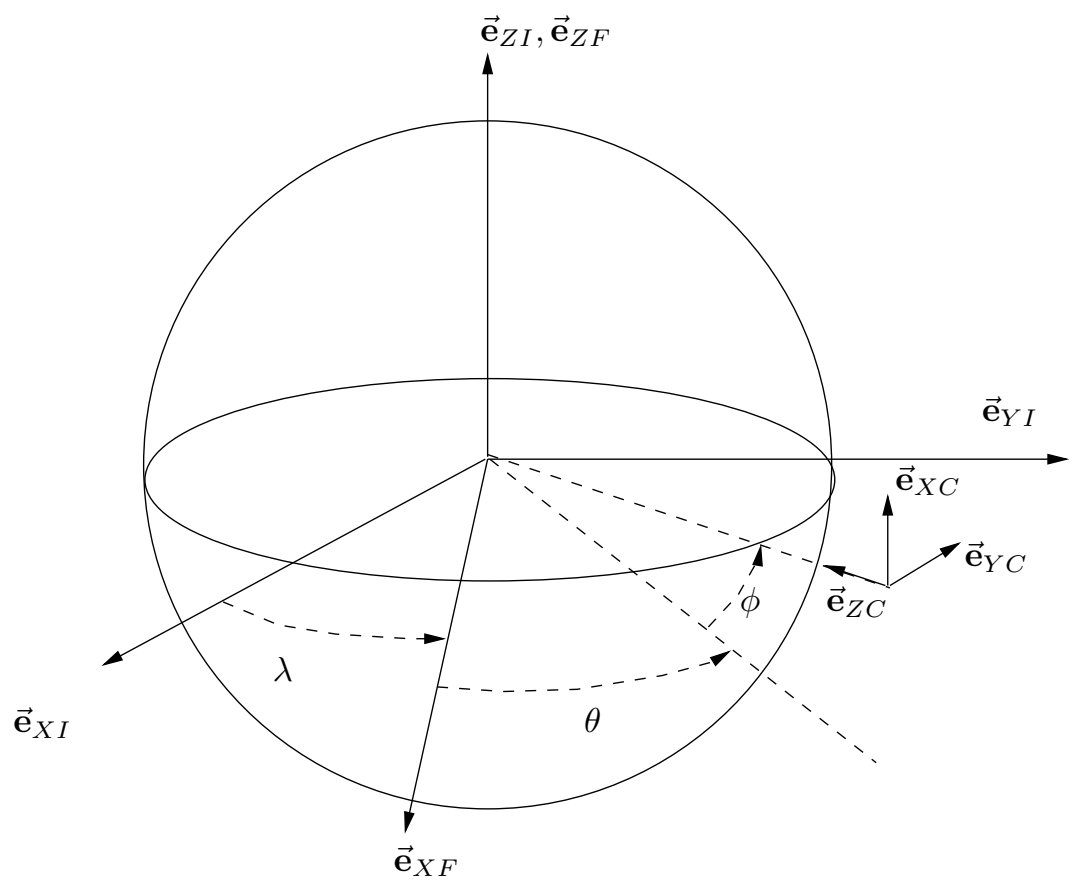

Figure 4. ECI, ECEF, and GC Frames

where $\epsilon=\phi_{g d}-\phi$, with $\phi_{g d}$ being the geodetic latitude of the vehicle's sub-latitude point, which is the projection of the vehicle position to the surface of the Earth along the $\overrightarrow{\mathbf{e}}_{Z D}$ axis. The geodetic latitude is then the angle between the $\overrightarrow{\mathbf{e}}_{Z D}$ axis and the equatorial plane of the Earth. The $G C$ and $G D$ frames are shown in Fig. 5.

An alternate measure of the location of the sub-latitude point is the geocentric latitude, $\phi_{g c}$, which is the angle from the equatorial plane of the Earth to the line connecting the sub-latitude point and the center of the Earth. Fig. 5 shows the differences between the declination, geocentric latitude, and geodetic latitude for a planet with exaggerated eccentricity. Note that the three angles are equal for a spherical planet. Fig. 5 also shows the physical meaning of the geodetic altitude, $h_{g d}$, and the magnitude of the radius vector, $r-$ two variables that will be used presently.

Table 1. Summary of WGS-84 Earth Model Constants

\begin{tabular}{lc}
\hline \hline Constant & Value \\
\hline$R_{\oplus}$ & $2.092565 \cdot 10^{7} \mathrm{ft}$ \\
$e_{\oplus}$ & $8.1819191 \cdot 10^{-2}$ \\
$\mu$ & $1.4076444 \cdot 10^{16} \mathrm{ft}^{3} / \mathrm{s}^{2}$ \\
$J_{2}$ & $1.0826230 \cdot 10^{-3}$ \\
$\Omega$ & $4.1780741 \cdot 10^{-3} \circ / \mathrm{s}$ \\
\hline \hline
\end{tabular}

The relationship between the geocentric and geodetic latitude is quite simple, and takes the form

$$
\tan \phi_{g c}=\left(1-e_{\oplus}^{2}\right) \tan \phi_{g d}
$$

The transformation between declination and geodetic latitude, however, is more complicated. Vallado ${ }^{16}$ gives the formulas

$$
\begin{aligned}
\tan \phi_{g d} & =\tan \phi+\frac{R_{\oplus} e_{\oplus}^{2} \sin \phi_{g d}}{r \cos \phi \sqrt{1-e_{\oplus}^{2} \sin ^{2} \phi_{g d}}} \\
h_{g d} & =\frac{r \cos \phi}{\cos \phi_{g d}}-\frac{R_{\oplus}}{\sqrt{1-e_{\oplus}^{2} \sin ^{2} \phi_{g d}}}
\end{aligned}
$$




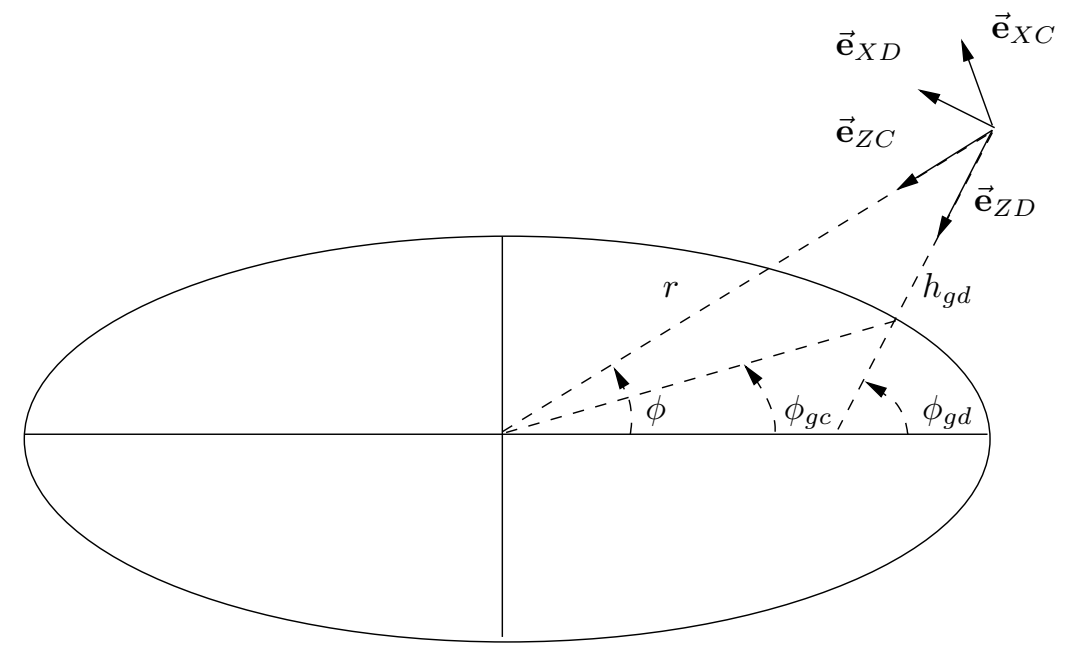

Figure 5. Declination, Geocentric Latitude, Geodetic Latitude, Geodetic Frame, and Geocentric Frame

Sofair ${ }^{17,18}$ determined an exact solution of Eqs. $(21-22), \phi_{g d}(r, \phi)$ and $h_{g d}(r, \phi)$. The solution is explicit, and is free of singularities at the poles and the equator, although the solution space is confined to a region outside a spheroid fixed at the center of the Earth with semimajor axis of approximately $43 \mathrm{~km}$. This restriction is of no concern for the present problem as the region is well within the surface of the Earth. The transformation of position from geodetic to geocentric coordinates is much less complicated, and may be determined directly from Eqs. (21) and (22). The WGS-84 values of $R_{\oplus}, e_{\oplus}$, and other constants that describe the shape, mass, and rotation of the Earth are summarized in Table 1.

Lastly, the body $(B)$ frame is fixed to the center of mass of the vehicle with the $\overrightarrow{\mathbf{e}}_{X B}$ axis directed through the nose of the vehicle, the $\overrightarrow{\mathbf{e}}_{Z B}$ axis oriented perpendicular to the $\overrightarrow{\mathbf{e}}_{X B}$ axis, in the plane of symmetry, with the positive direction taken to be toward the bottom of the vehicle, and the $\overrightarrow{\mathbf{e}}_{Y B}$ axis completing the righthanded system so that it points out the right side of the vehicle. The $B$ frame is shown in Fig. 3 . The orientation of the $B$ frame is typically expressed by a 3-2-1 Euler angle sequence relating the $G D$ frame to the $B$ frame. The transformation is given by

$$
\begin{aligned}
\left\{\begin{array}{l}
\overrightarrow{\mathbf{e}}_{X B} \\
\overrightarrow{\mathbf{e}}_{Y B} \\
\overrightarrow{\mathbf{e}}_{Z B}
\end{array}\right\} & =\mathbf{G}_{G D 2 B}\left\{\begin{array}{c}
\overrightarrow{\mathbf{e}}_{X D} \\
\overrightarrow{\mathbf{e}}_{Y D} \\
\overrightarrow{\mathbf{e}}_{Z D}
\end{array}\right\} \\
\mathbf{G}_{G D 2 B} & =\left[\begin{array}{ccc}
1 & 0 & 0 \\
0 & \cos \Phi & \sin \Phi \\
0 & -\sin \Phi & \cos \Phi
\end{array}\right] \cdot\left[\begin{array}{ccc}
\cos \Theta & 0 & -\sin \Theta \\
0 & 1 & 0 \\
\sin \Theta & 0 & \cos \Theta
\end{array}\right] \cdot\left[\begin{array}{ccc}
\cos \Psi & \sin \Psi & 0 \\
-\sin \Psi & \cos \Psi & 0 \\
0 & 0 & 1
\end{array}\right]
\end{aligned}
$$

where $\Psi, \Theta$, and $\Phi$ are the azimuth, elevation, and bank angles, respectively, also commonly referred to as the yaw, pitch, and roll angles.

Although the Euler angle sequence offers an ease of visualization, other parameterizations of the vehicle orientation are preferable for implementation in the equations of motion and filtering algorithms. ${ }^{20}$ The most advantageous of these parameterizations are the Euler parameters, which are defined in terms of the Euler angles as ${ }^{20}$

$$
\begin{aligned}
& e_{0}=\cos \left(\frac{\Phi}{2}\right) \cos \left(\frac{\Theta}{2}\right) \cos \left(\frac{\Psi}{2}\right)+\sin \left(\frac{\Phi}{2}\right) \sin \left(\frac{\Theta}{2}\right) \sin \left(\frac{\Psi}{2}\right) \\
& e_{1}=\sin \left(\frac{\Phi}{2}\right) \cos \left(\frac{\Theta}{2}\right) \cos \left(\frac{\Psi}{2}\right)-\cos \left(\frac{\Phi}{2}\right) \sin \left(\frac{\Theta}{2}\right) \sin \left(\frac{\Psi}{2}\right) \\
& e_{2}=\cos \left(\frac{\Phi}{2}\right) \sin \left(\frac{\Theta}{2}\right) \cos \left(\frac{\Psi}{2}\right)+\sin \left(\frac{\Phi}{2}\right) \cos \left(\frac{\Theta}{2}\right) \sin \left(\frac{\Psi}{2}\right) \\
& e_{3}=\cos \left(\frac{\Phi}{2}\right) \cos \left(\frac{\Theta}{2}\right) \sin \left(\frac{\Psi}{2}\right)-\sin \left(\frac{\Phi}{2}\right) \sin \left(\frac{\Theta}{2}\right) \cos \left(\frac{\Psi}{2}\right)
\end{aligned}
$$


The use of Euler parameters leads to a representation of the attitude transformation matrix of the form

$$
\mathbf{G}_{G D 2 B}=\left[\begin{array}{ccc}
e_{0}^{2}+e_{1}^{2}-e_{2}^{2}-e_{3}^{2} & 2\left(e_{1} e_{2}+e_{0} e_{3}\right) & 2\left(e_{1} e_{3}-e_{0} e_{2}\right) \\
2\left(e_{1} e_{2}-e_{0} e_{3}\right) & e_{0}^{2}-e_{1}^{2}+e_{2}^{2}-e_{3}^{2} & 2\left(e_{0} e_{1}+e_{2} e_{3}\right) \\
2\left(e_{1} e_{3}+e_{0} e_{2}\right) & 2\left(e_{2} e_{3}-e_{0} e_{1}\right) & e_{0}^{2}-e_{1}^{2}-e_{2}^{2}+e_{3}^{2}
\end{array}\right]
$$

The Euler parameters constitute a four parameter set that describe the orientation of the $B$ frame with respect to the $G D$ frame. Due to the fact that four parameters are used to describe a rotation with three degrees of freedom, the constraint equation $e_{0}^{2}+e_{1}^{2}+e_{2}^{2}+e_{3}^{2}=1$ must also be satisfied.

\section{Equations of Motion}

Newton's laws of motion, expressed for a body moving relative to a planet-fixed reference frame, lead to the dynamic equation

$$
\ddot{\overrightarrow{\mathbf{r}}}+2 \overrightarrow{\boldsymbol{\Omega}} \times \dot{\overrightarrow{\mathbf{r}}}+\overrightarrow{\boldsymbol{\Omega}} \times \overrightarrow{\boldsymbol{\Omega}} \times \overrightarrow{\mathbf{r}}=\frac{\overrightarrow{\mathbf{F}}}{m}
$$

The quantity $\overrightarrow{\mathbf{r}}$ may be expressed in the $G C$ frame as $\overrightarrow{\mathbf{r}}=-r \overrightarrow{\mathbf{e}}_{Z C}$. The velocity and acceleration follow as

$$
\begin{aligned}
\dot{\overrightarrow{\mathbf{r}}}= & r \dot{\phi} \overrightarrow{\mathbf{e}}_{X C}+r \dot{\theta} \cos \phi \overrightarrow{\mathbf{e}}_{Y C}-\dot{r} \overrightarrow{\mathbf{e}}_{Z C} \\
\ddot{\overrightarrow{\mathbf{r}}=} & \left(r \ddot{\phi}+2 \dot{r} \dot{\phi}+r \dot{\theta}^{2} \sin \phi \cos \phi\right) \overrightarrow{\mathbf{e}}_{X C}+(r \ddot{\theta} \cos \phi+2 \dot{r} \dot{\theta} \cos \phi-2 r \dot{\phi} \dot{\theta} \sin \phi) \overrightarrow{\mathbf{e}}_{Y C}- \\
& \left(\ddot{r}-r \dot{\phi}^{2}-r \dot{\theta}^{2} \cos ^{2} \phi\right) \overrightarrow{\mathbf{e}}_{Z C}
\end{aligned}
$$

The angular velocity of the planet may be expanded as $\overrightarrow{\boldsymbol{\Omega}}=\Omega\left(\cos \phi \overrightarrow{\mathbf{e}}_{X C}-\sin \phi \overrightarrow{\mathbf{e}}_{Z C}\right)$.

The force per unit mass acting on the vehicle, including the second zonal harmonic is given by

$$
\frac{\overrightarrow{\mathbf{F}}}{m}=\left(a_{X C}-\frac{3 \mu J_{2}}{2 r^{4}} \sin 2 \phi\right) \overrightarrow{\mathbf{e}}_{X C}+a_{Y C} \overrightarrow{\mathbf{e}}_{Y C}+\left(a_{Z C}+\frac{\mu}{r^{2}}-\frac{3 \mu J_{2}}{2 r^{4}}\left(2-3 \cos ^{2} \phi\right)\right) \overrightarrow{\mathbf{e}}_{Z C}
$$

where $a_{X C}, a_{Y C}$, and $a_{Z C}$ are the components of the geocentric representation of the applied acceleration.

$$
\begin{aligned}
\dot{r} & =-w \\
\dot{\phi} & =\frac{u}{r} \\
\dot{\theta} & =\frac{v}{r \cos \phi}-\Omega \\
\dot{u} & =a_{X C}+\frac{1}{r}\left(u w-v^{2} \tan \phi\right)-\frac{3 \mu J_{2}}{2 r^{4}} \sin (2 \phi) \\
\dot{v} & =a_{Y C}+\frac{1}{r}(u v \tan \phi+v w) \\
\dot{w} & =a_{Z C}-\frac{1}{r}\left(u^{2}+v^{2}\right)+\frac{\mu}{r^{2}}-\frac{3 \mu J_{2}}{2 r^{4}}\left(2-3 \cos ^{2} \phi\right)
\end{aligned}
$$

where $u, v$, and $w$ are the $x, y$, and $z$-axis components of the velocity in the $G C$ frame, respectively, and $a_{X C}, a_{Y C}, a_{Z C}$ are the geocentric representation of the accelerations acting at the body center of mass, not including the gravitational terms.

The sensed accelerations at the vehicle center of gravity (denoted by $a_{X B}, a_{Y B}$, and $a_{Z B}$ ) are transformed into the $G C$ frame to be consistent with the equations of motion by means of

$$
\left\{\begin{array}{c}
a_{X C} \\
a_{Y C} \\
a_{Z C}
\end{array}\right\}=\mathbf{G}_{B 2 G C}\left\{\begin{array}{c}
a_{X B} \\
a_{Y B} \\
a_{Z B}
\end{array}\right\}
$$

where $\mathbf{G}_{B 2 G C}=\left(\mathbf{G}_{G D 2 B} \mathbf{G}_{G C 2 G D}\right)^{T}$.

The rotational kinematics of a vehicle relative to the $G D$ frame may be written as

$$
\left\{\begin{array}{c}
\dot{e}_{0} \\
\dot{e}_{1} \\
\dot{e}_{2} \\
\dot{e}_{3}
\end{array}\right\}=\frac{1}{2}\left[\begin{array}{ccc}
-e_{1} & -e_{2} & -e_{3} \\
e_{0} & -e_{3} & e_{2} \\
e_{3} & e_{0} & -e_{1} \\
-e_{2} & e_{1} & e_{0}
\end{array}\right]\left(\left\{\begin{array}{c}
P \\
Q \\
R
\end{array}\right\}-\frac{1}{r} \mathbf{G}_{G C 2 B}\left\{\begin{array}{c}
v \\
-u \\
-v \tan \phi
\end{array}\right\}\right)
$$


Note that the $B$ frame accelerations at the IMU location $a_{X T}, a_{Y T}, a_{Z T}$ and the $B$ frame inertial angular rates, $P, Q$, and $R$ are assumed to be known functions of time. The next section discusses the application of the filtering and smoothing methods to the Hyper-X Mach 10 test flight.

\section{Application to the Hyper-X Mach 10 Flight}

This section summarizes the results of applying the methods developed in the previous sections to the problem of estimating the trajectory of the Hyper-X vehicle on its third test flight, which occurred on November 16, 2004. This test flight was designed to reach a test condition of approximately Mach 9.6. The flight data preprocessing methods are discussed first.

\section{A. Flight Data Preprocessing}

This section summarizes the data preprocessing that were required in order to provide useful measurements as needed for the trajectory reconstruction process. The raw flight data were passed through a preprocessing tool, known as PREPR, ${ }^{21}$ which consists of four main utilities. The first utility removes duplicate points in the data series which result from differences between the sensor data refresh rate and the telemetry sampling rate. The second utility allows users to interactively remove rogue points from the data series. Users select individual or groups of points to remove from a graph of the data series. The third utility samples the data series at a uniform time step, using a user-specified option of linear, cubic spline, or Hermite spline interpolation between available data points. The fourth utility gives the user the option to filter the data series using Fourier series and optimal Weiner filtering method ${ }^{22}$ available within the Systems Identification Program for Aircraft (SIDPAC). ${ }^{23}$ A separate fifth utility is included which can be used to differentiate a data series once it has been passed through the previous four routines. The user is given the option to use backward difference or center divided difference methods. Alternatively, users can select to curve fit the data using either cubic or Hermite spline polynomials, and to individually differentiate each piecewise segment.

\section{Inertial Data}

Measurements of acceleration and angular rates were provided by QA2000 accelerometers and GG1320 Digital Laser Gyroscopes (DLG) contained within the Honeywell H-764G system, ${ }^{24}$ a unit with a background of numerous civilian and military applications. These measurement data were obtained from the on-board telemetry stream at a rate of $100 \mathrm{~Hz}$ and were first preprocessed by removing points which contained obviously bad or rogue data. Next, the data were edited to remove points which could be considered as repeated, stale, or non-updated frames. Gaps in the data due to telemetry dropouts or due to edited data were filled in by a piecewise cubic Hermite interpolating function, and were then filtered to remove the effects of high-frequency structural noise, measurement noise, and to smooth out the data from the effects of sensor quantization. Numerical derivatives of the inertial angular rates, as required for evaluation of the equations of motion, were calculated using a second-order centered divided difference scheme, except for the beginning and end points, which used first-order forward and backward divided differences, respectively.

An example of raw data and processed inertial flight data is shown in Fig. 6, which shows the pitch rate ( $B$-frame $y$-axis angular velocity measurement) during boost. The raw signal shows the evidence of a known $8 \mathrm{~Hz}$ structural mode that was eliminated in the course of data pre-processing.

Values of the process noise standard deviations used in connection with the inertial measurement data in the EKF formulation were taken from the Honeywell specifications given in Ref. 25. The inertial measurement data were also corrected for misalignment between the IMU mounting and the vehicle $B$ frame, measured before flight and given in Ref. 26. Uncertainties in the preflight mounting measurements were provided and were taken into consideration when computing the uncertainties in the trajectory estimate.

\section{GPS Data}

GPS measurements were obtained as outputs from the H-764G GPS Embedded Module (GEM), which is a 5-channel receiver, operating in Precision Positioning Service (PPS) mode at a rate of $1 \mathrm{~Hz}$. The GPS data required little preprocessing, aside from extracting the data from the telemetry stream. There were several issues with the data itself, however. First, the east velocity channel saturated at approximately 27 sec. after the HXLV was dropped from the B-52 carrier vehicle, due to inadequate telemetry system bit assignments. The east velocity data unsaturated during the vehicle descent, at a time of approximately 688 sec. after drop. This saturation was a known problem and is due only to telemetry and is not a problem with the $\mathrm{H}-764 \mathrm{G}$ system. 


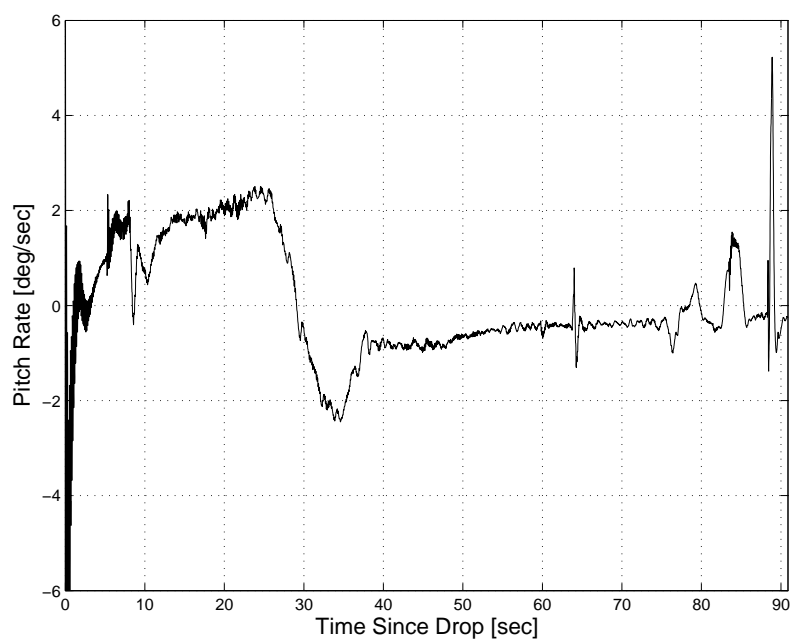

(a) Raw Pitch Rate

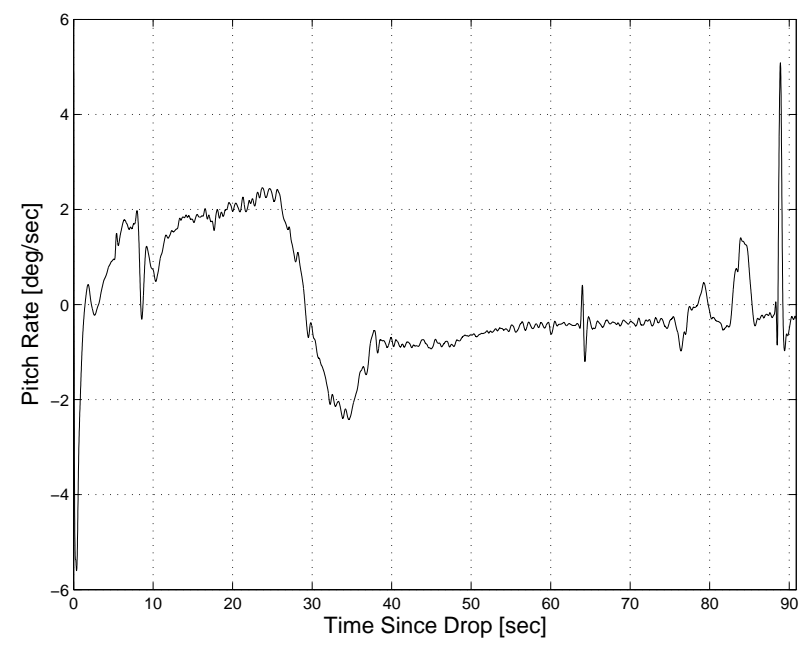

(b) Processed Pitch Rate

Figure 6. Comparison of Raw and Processed Pitch Rate During Boost

A second concern is that the GPS antenna carried on the HXRV itself was activated at the instant of the drop (previous GPS points from the HXRV telemetry stream were provided by the vehicle flight management computer, but were derived from a GPS antenna carried on the B-52), and as a result, suspected blockage or multi path effects may have corrupted the first several data points recorded after the drop. Therefore, the first several points were deleted from the data set and were not considered in the reconstruction process. Also, the GPS receiver lost lock on all satellites toward the end of the descent trajectory at a time of approximately 755 seconds after drop. The lock was regained at 780 seconds. During this period there were no substantial roll oscillations and the cause for the loss of lock is currently unknown. The data points in the telemetry stream during this time interval were removed from consideration for trajectory reconstruction purposes.

\section{B. Atmosphere Reconstruction}

The atmosphere reconstruction for the third flight of the Hyper-X vehicle is based on weather balloon data acquired both before and after the actual test flight. The atmosphere reconstruction is described in Ref. 27. The anchor point for the atmosphere reference was $\phi_{g d_{r e f}}=33.45^{\circ}, \theta_{r e f}=-121.0667^{\circ}$. Weather data at a point other than the location of the reference point must be transformed by making use of pressure gradient information to map the pressure to such a location. The temperature table is assumed to be invariant with respect to latitude and longitude, and the density follows directly from the application of the equation of state.

The vehicle ground track relative to the atmosphere reference point is shown in Fig. 7. The drop, separation, and loss of signal points are also shown for reference. Clearly the evaluation of atmospheric data along the trajectory requires a large extrapolation from the region in which the data were originally captured, which represents a significant source of uncertainty.

\section{Results of the Reconstruction}

The results of the trajectory reconstruction for the Mach 10 flight of the Hyper-X vehicle is discussed in this section. The comparison of RMS errors between the reconstructed solution and the inertial solution with the GPS measurements is shown in Table 2. In all cases the trajectory reconstruction is a better fit for the GPS data than the pure inertial solution, as would be expected.

An example of the improvement in the trajectory estimate gained by using the Kalman filter reconstruction method can be seen in Fig. 8. This figure shows the north velocity solution during the boost phase, in which the reconstructed solution is shown in the solid curve, the on-board inertial solution is shown in the dashed curve, and the individual GPS solutions are shown with the square points. The drift the in the inertial solution is apparent, but is seen clearly in the residual plot, which shows the north velocity residuals during boost. The inertial solution residual shows a clear trend toward a value of approximately $1 \mathrm{ft} / \mathrm{sec}$ while the residuals of the reconstructed solution show no trend or bias. The maximum residual of the inertial 


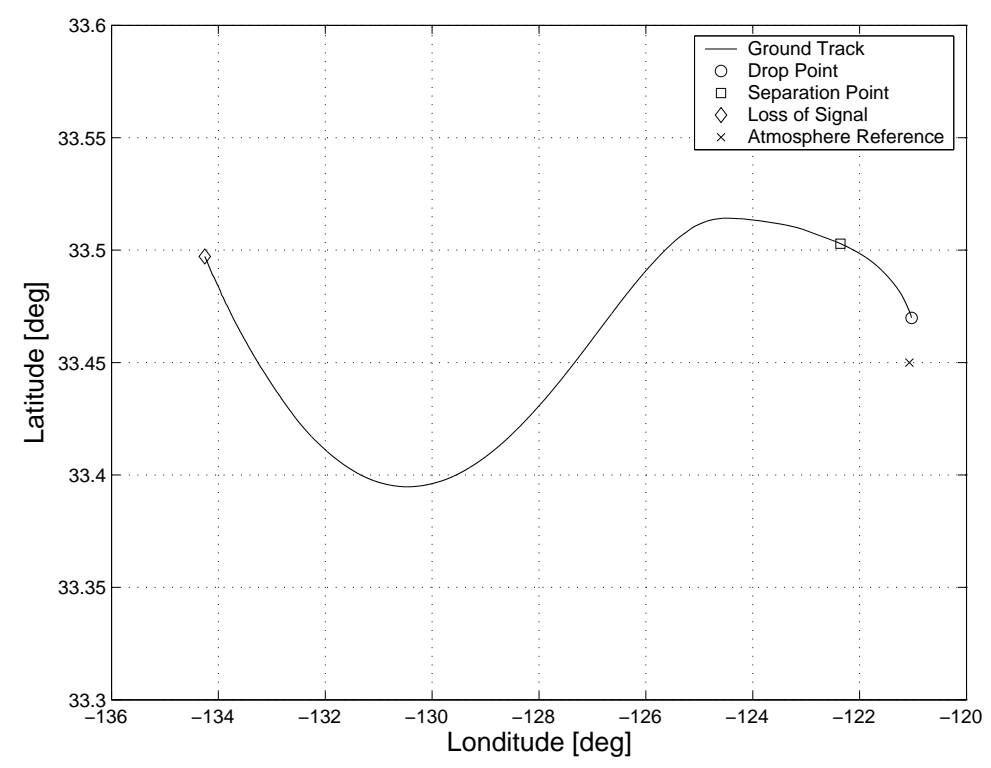

Figure 7. Ground Track Relative to Atmosphere Reference

Table 2. Summary of RMS Errors

\begin{tabular}{lcccc}
\hline \hline Measurement & EKF & NAV & Ratio & Observations \\
\hline$h_{g d}, \mathrm{ft}$ & 33.52 & 35.51 & 1.059 & 710 \\
$\phi_{g d}, \mathrm{deg}$ & $2.415 \cdot 10^{-5}$ & $7.890 \cdot 10^{-4}$ & 32.67 & 710 \\
$\theta, \mathrm{deg}$ & $3.006 \cdot 10^{-4}$ & $1.441 \cdot 10^{-3}$ & 4.794 & 710 \\
$u_{g d}, \mathrm{ft} / \mathrm{sec}$ & 0.3979 & 0.6425 & 1.615 & 710 \\
$v_{g d}, \mathrm{ft} / \mathrm{sec}$ & 0.8183 & 1.300 & 1.589 & 104 \\
$w_{g d}, \mathrm{ft} / \mathrm{sec}$ & 0.7546 & 0.8374 & 1.110 & 710 \\
\hline \hline
\end{tabular}

solution is small compared with the magnitude of the total velocity, but nevertheless it is a systematic error that has been compensated for by using the filter/smoother approach presented in this paper.

The next several subsections discuss the trajectory reconstruction and some comparisons with the onboard pure inertial solution during the boost, separation, and descent phases of the flight. Comparison against the preflight nominal trajectory and Monte-Carlo error bands are also shown.

\section{Boost Trajectory}

The altitude, Mach number, dynamic pressure, and angle of attack time histories during the boost phase of the trajectory are shown in Fig. 9. Here, the preflight Monte-Carlo average trajectory is shown in the dashed-dot line type, the Monte-Carlo +/- 3 standard deviations away from the nominal trajectory are shown in the dashed line type, the best-estimated trajectory is shown in the solid line type, and the +/3 standard deviations of knowledge uncertainty are shown in the dotted line type. The pre-flight boost Monte-Carlo simulation is discussed in Ref. 4.

Clearly these data indicate that the altitude and Mach profiles are well within the pre-flight predictions. The dynamic pressure is initially below the $3 \sigma$ pre-flight prediction, but the difference between the BET and the Monte-Carlo average rapidly decreases and for the remainder of the boost trajectory the dynamic pressure is essentially nominal. The initial deviation between the nominal dynamic pressure and reconstructed dynamic pressure is due to a difference in the range-reference atmosphere and the reconstructed atmosphere as well as a difference in the nominal and reconstructed velocity.

The angle of attack time history starts near nominal but deviates briefly beyond the $+/-3$ Monte-Carlo standard deviations in the transonic region and in the initial drop to the negative angle of attack region. 


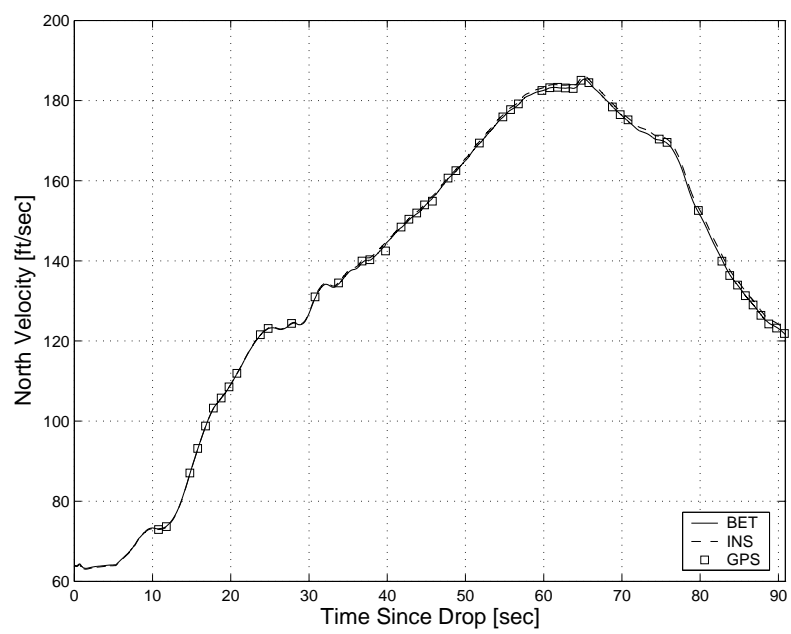

(a) North Velocity

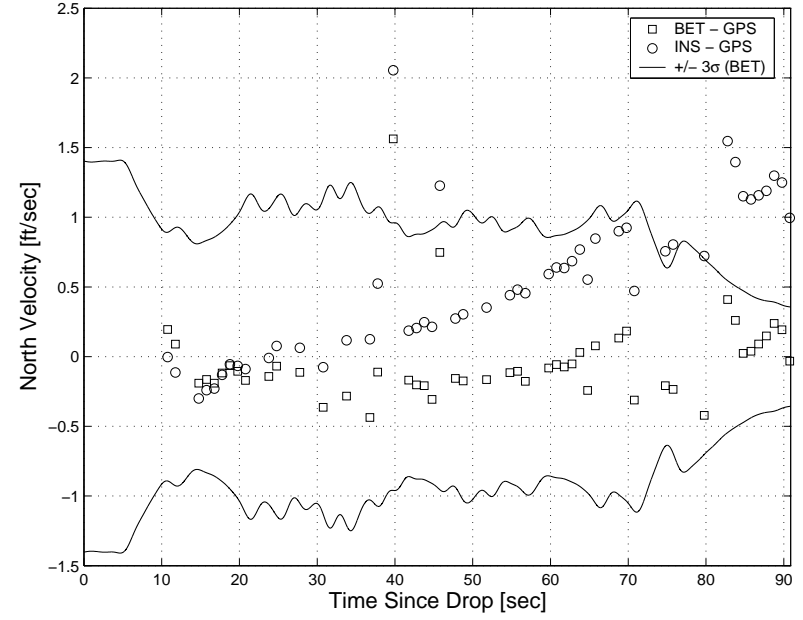

(b) North Velocity Residual

Figure 8. North Velocity During Boost

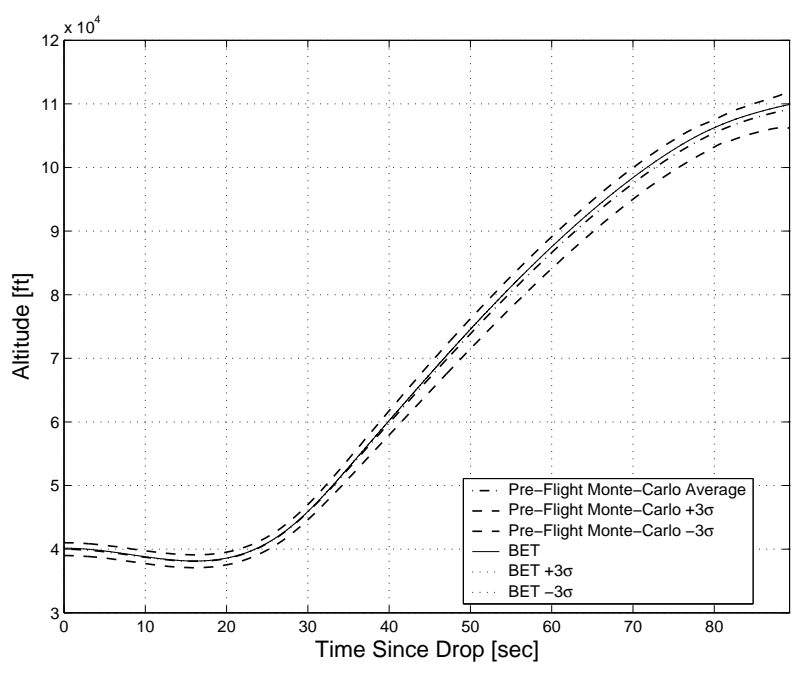

(a) Altitude

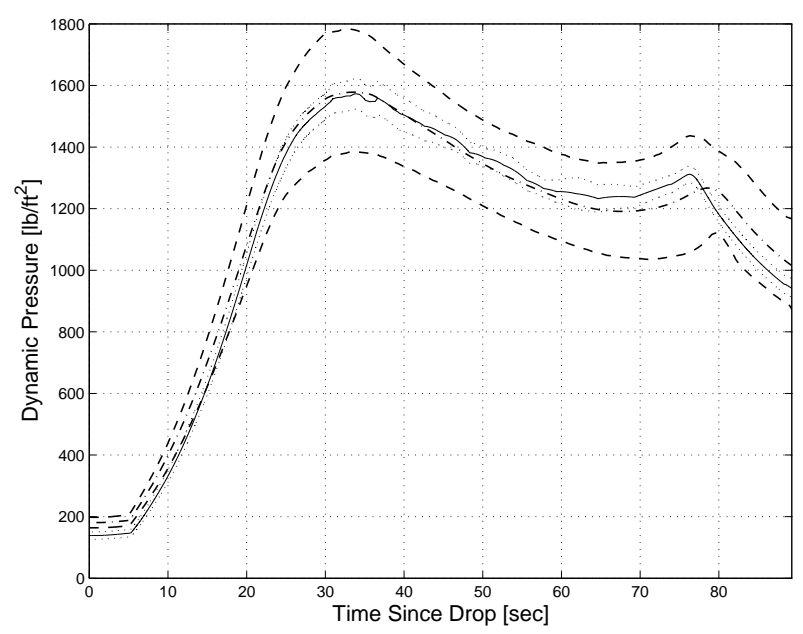

(c) Dynamic Pressure

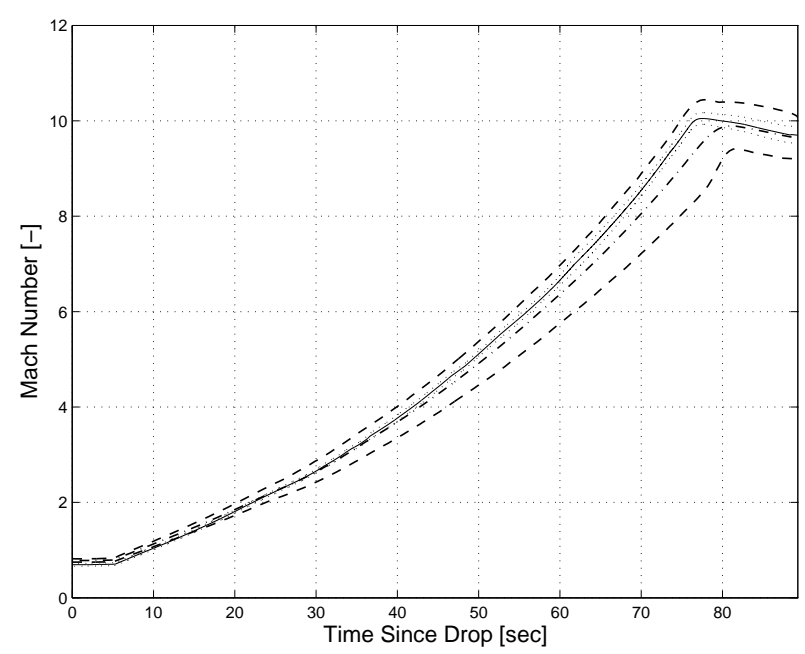

(b) Mach Number

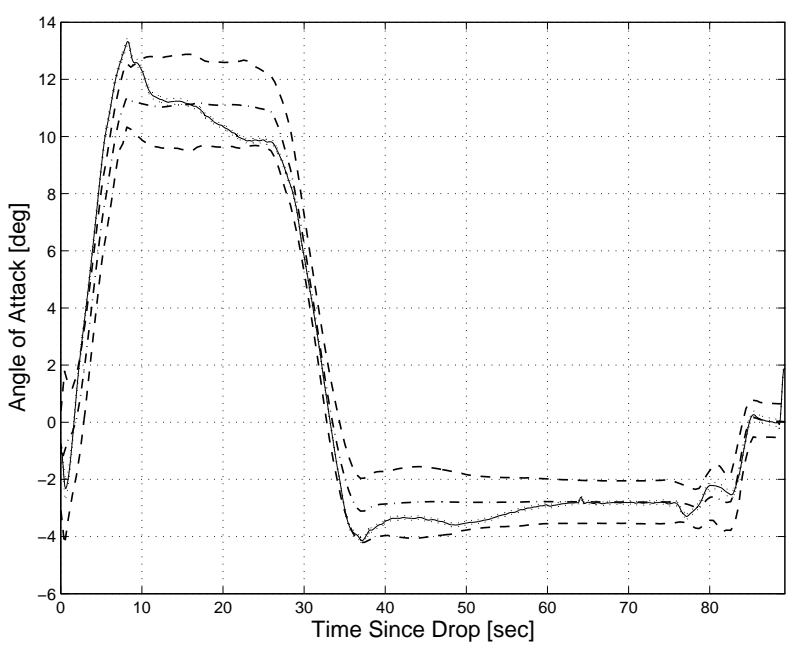

(d) Angle of Attack

Figure 9. Trajectory Data During Boost 


\section{Separation}

The conditions at the separation between the HXLV and the HXRV are summarized in Table 3 . The time of the commanded separation is $88.35 \mathrm{sec}$ after the sensed drop time. The table lists the target separation condition and the range of acceptable deviation as specified in Ref. 28, along with the separation condition as indicated by the inertial navigation system (INS) with the day-of-flight atmosphere reconstruction. The last two columns give the separation condition determined by the trajectory reconstruction (BET) and the associated $1 \sigma$ uncertainties.

Table 3. Flight 3 Separation Conditions

\begin{tabular}{ccccc}
\hline \hline Variable & Target Requirements & INS & BET & BET Uncertainty \\
\hline Time Since Drop, sec & 104.0 (Max) & 88.35 & 88.35 & 0.0 \\
Altitude, ft & 109,580 & 109,601 & 109,653 & 6.4 \\
Mach number & $9.6 \pm 0.6$ & 9.703 & 9.702 & 0.057 \\
Dynamic Pressure, lb/ft ${ }^{2}$ & $1000 \pm 210.0$ & 957 & 954 & 9.9 \\
Flight Path Angle, deg & $1.5 \pm 1.0$ & 1.62 & 1.66 & 0.08 \\
Angle of Attack, deg & $0.0 \pm 1.5$ & -0.028 & -0.011 & 0.043 \\
Angle of Sideslip, deg & $0.0 \pm 1.0$ & -0.20 & -0.20 & 0.054 \\
Roll Angle, deg & $0.0 \pm 15.0$ & 1.72 & 1.67 & 0.16 \\
Pitch Rate, deg/sec & $-0.18 \pm 3.0$ & 0.0027 & 0.414 & 0.005 \\
Yaw Rate, deg/sec & $0.0 \pm 3.0$ & 0.304 & 0.292 & 0.005 \\
Roll Rate, deg/sec & $0.0 \pm 6.0$ & 0.032 & -0.05 & 0.005 \\
\hline \hline
\end{tabular}

Trajectory data during the separation of the vehicles is shown in Fig. 10. Similar to the boost trajectory data shown earlier, the pre-flight Monte-Carlo average trajectory is shown in the dashed-dot line type, the Monte-Carlo +/- 3 standard deviations away from the nominal trajectory are shown in the dashed line type, the best-estimated trajectory is shown in the solid line type, and the $+/-3$ standard deviations of knowledge uncertainty are shown in the dotted line type. The Monte-Carlo separation simulation is discussed in Ref. 29 and background information on the stage separation system with flight data evaluation is provided in Ref. 30. All trajectory data are well within the pre-flight predictions, indicating an essentially perfect separation between the launch vehicle and the research vehicle.

\section{Engine Test Trajectory}

The scramjet engine test phase of the mission began with the opening of the engine cowl door at a time of approximately $2.5 \mathrm{sec}$. after the separation of the HXRV from the HXLV. The engine was fuelled for approximately $10 \mathrm{sec}$. The vehicle achieved a maximum Mach number during the scramjet engine test of approximately 9.6. The trajectory during this phase of the mission was again extremely close to the preflight predictions, but may not be shown due to sensitivity concerns. The scramjet engine test, including post-flight comparison with the pre-flight predictions, is described in Ref. 31.

\section{Descent Trajectory}

After completion of the scramjet engine test, the engine cowl door was closed and the vehicle then executed a pull-up maneuver to slow the descent. After completion of this maneuver, the vehicle was commanded to follow a descent trajectory that included a series of parameter identification maneuvers at each Mach number occurring from 8 to 2. The design and analysis of these parameter identification maneuvers is discussed in Refs. 32 and 33.

The reconstructed trajectory data during the descent is shown in Fig. 11. Again, the pre-flight MonteCarlo average trajectory is shown in the dashed-dot line type, the Monte-Carlo +/- 3 standard deviations away from the nominal trajectory are shown in the dashed line type, the best-estimated trajectory is shown in the solid line type, and the +/- 3 standard deviations of knowledge uncertainty are shown in the dotted line type. The trajectory data are, for the most part, well within the pre-flight Monte-Carlo bounds. The main exception is the roll angle time history. Shortly after the closing of the engine cowl door the vehicle experienced some rocking motion. The cause of this motion is still under investigation. ${ }^{32}$ 


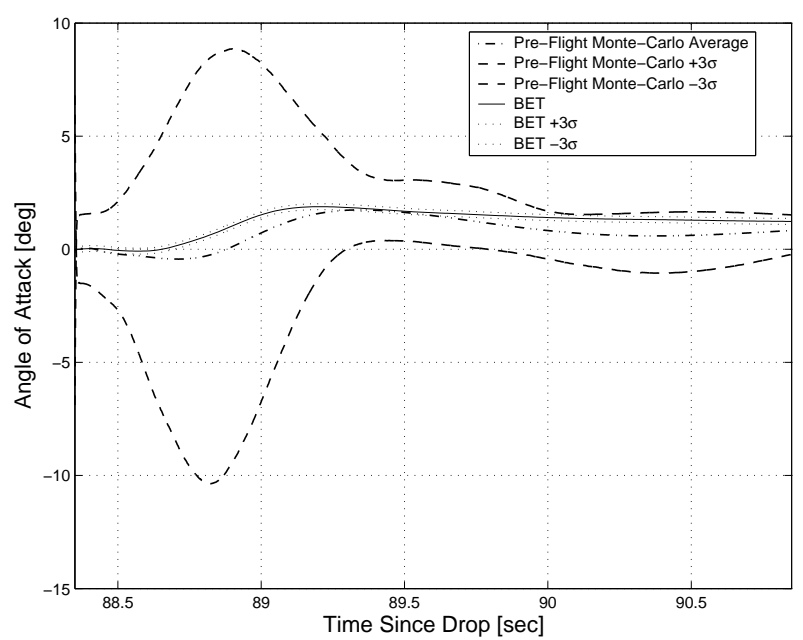

(a) Angle of Attack

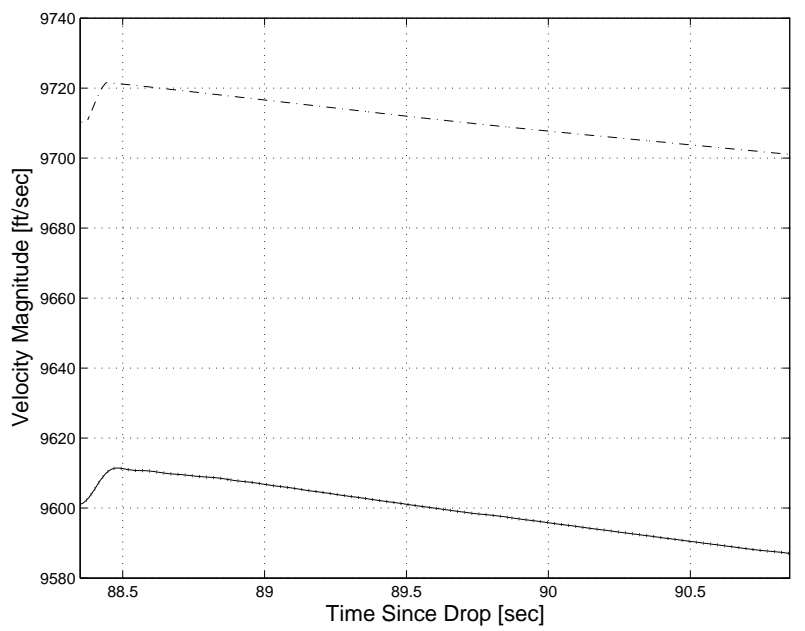

(c) Velocity Magnitude

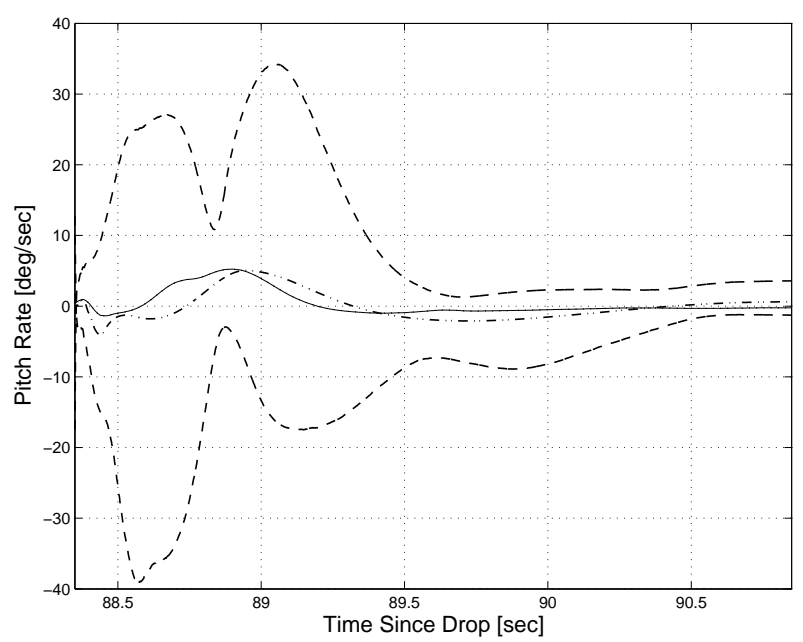

(e) Pitch Rate

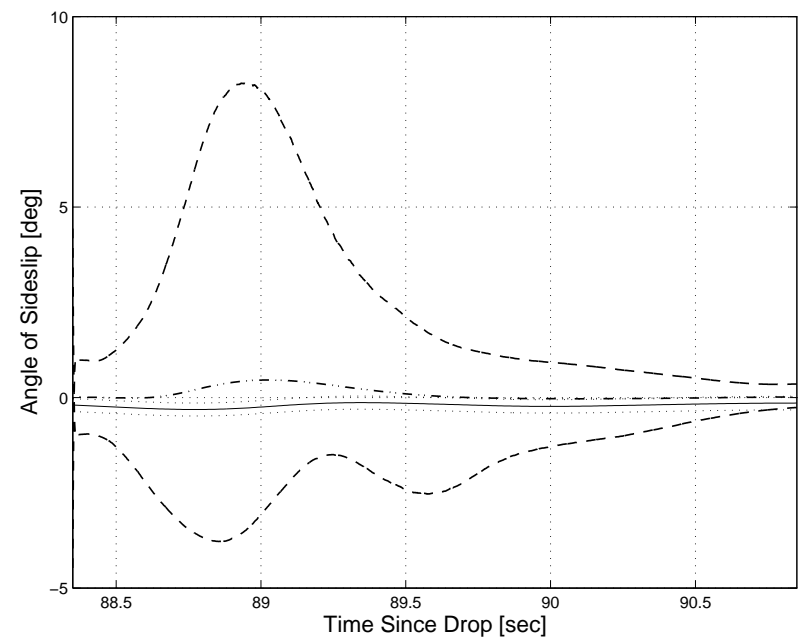

(b) Angle of Sideslip

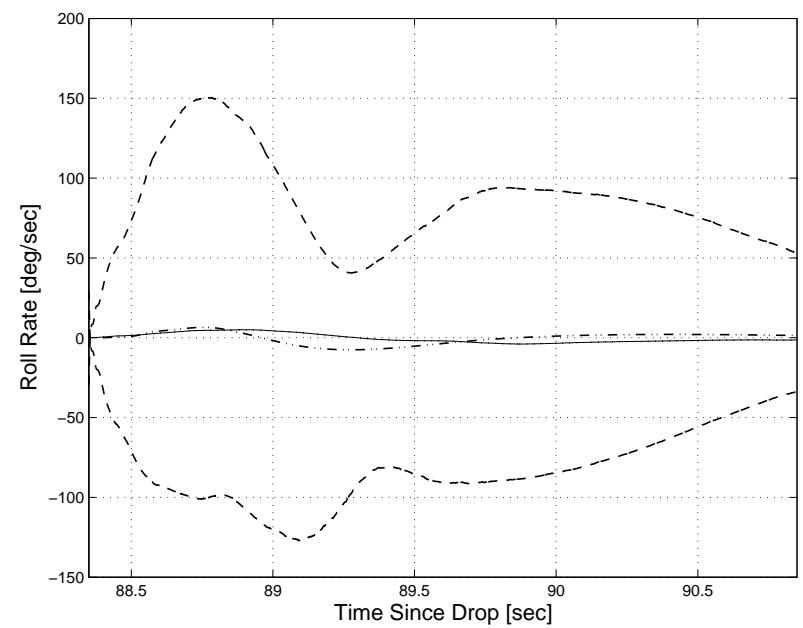

(d) Roll Rate

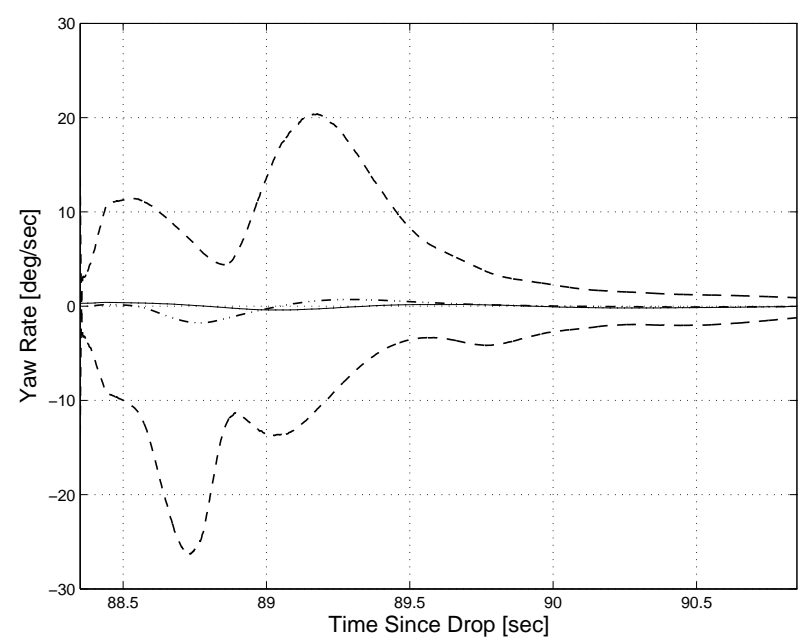

(f) Yaw Rate

Figure 10. Trajectory Data During Separation 


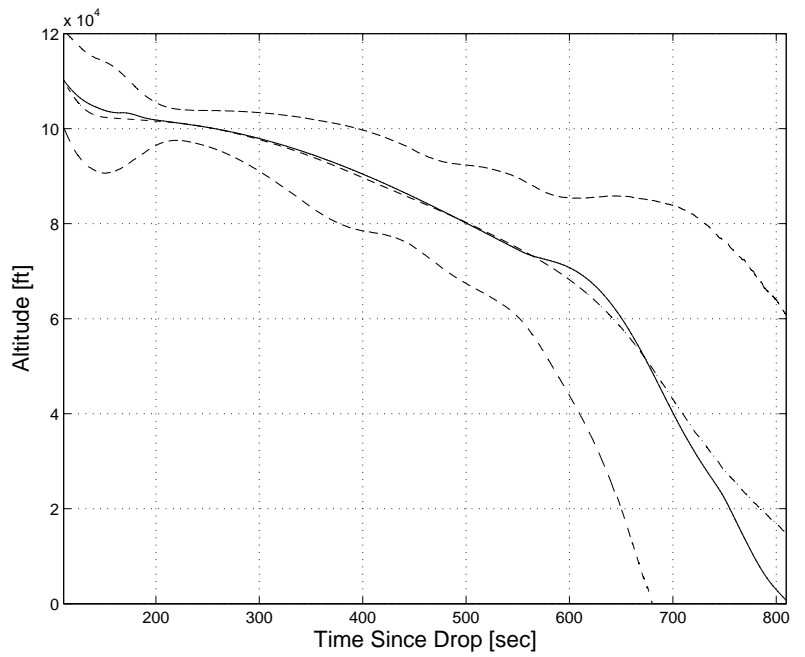

(a) Altitude

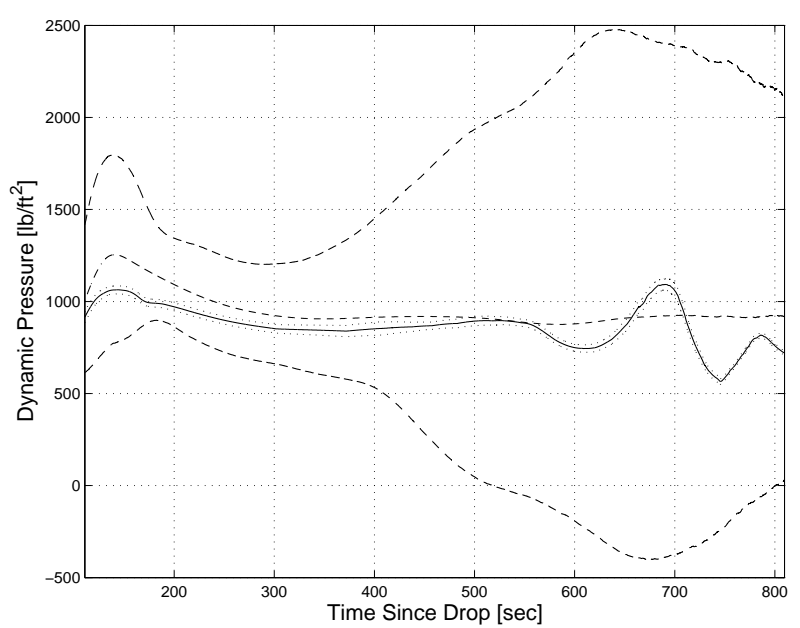

(c) Dynamic Pressure

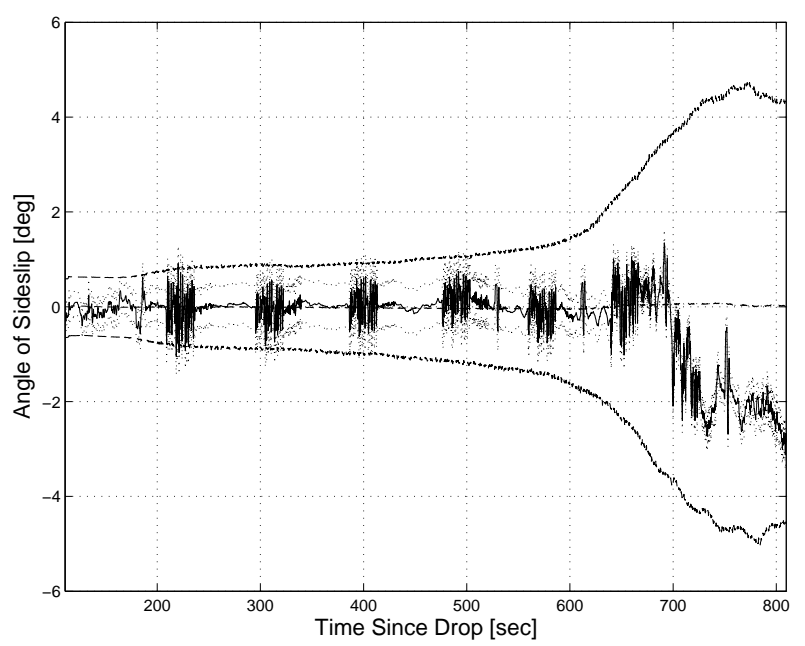

(e) Angle of Sideslip

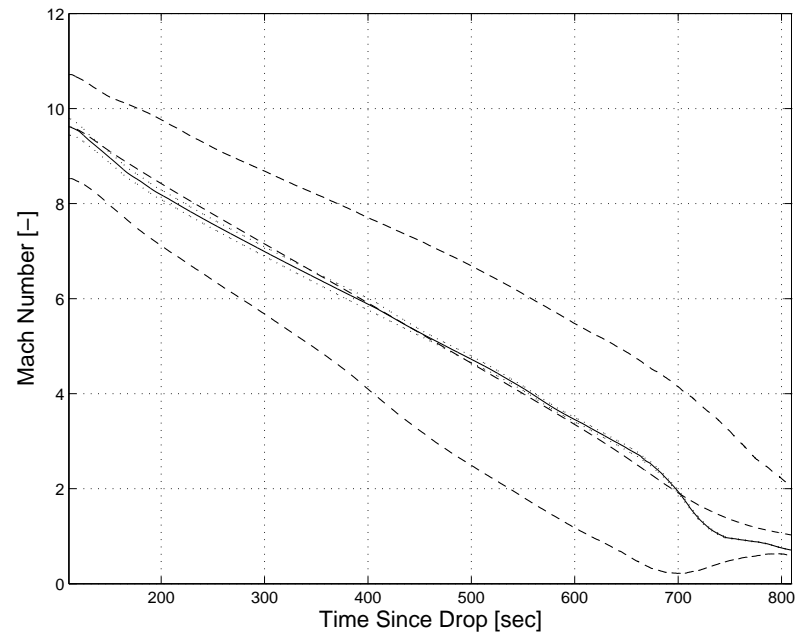

(b) Mach Number

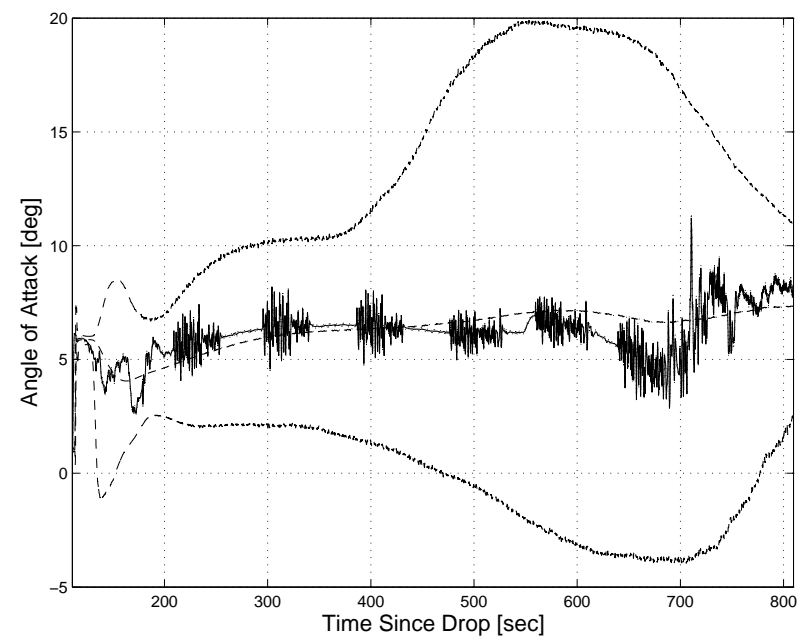

(d) Angle of Attack

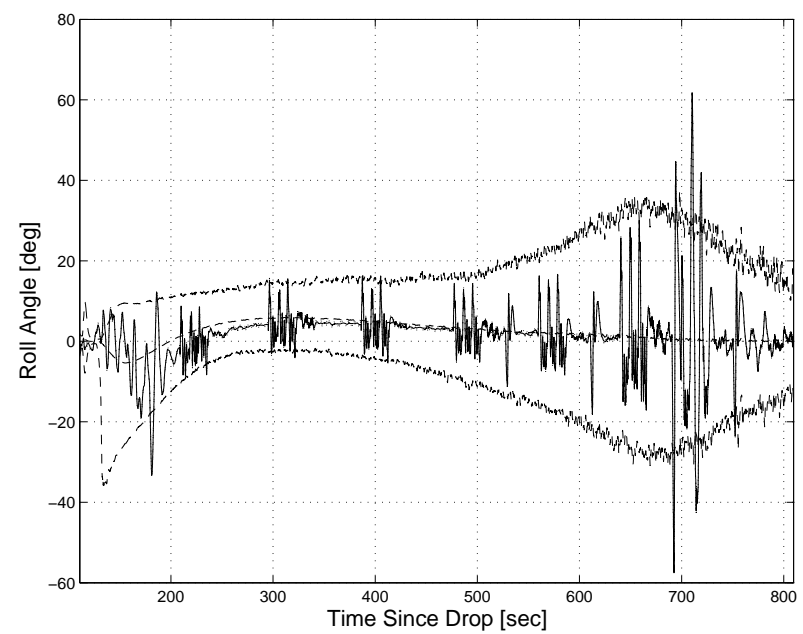

(f) Roll Angle

Figure 11. Trajectory Data During Descent 


\section{Conclusions}

This paper has discussed the formulation and implementation of an Extended Kalman Filtering algorithm for use in reconstructing the flight path of the Hyper-X/X-43A vehicle, using in-flight inertial measurements in the dynamic model combined with redundant measurements of the vehicle state from independent sources, particularly from the Global Positioning System. The equations of motion were formulated in a local, noninertial coordinate system, and included the effects of several systematic error sources. Equations relating the state to measured quantities were provided for a variety of data sources of interest to this problem. Data recorded during the Hyper-X Mach 10 flight on November 16, 2004, were first preprocessed and then processed using the extended Kalman filter, leading to an improvement over the on-board navigation solution. Results for the boost, separation, and descent phases of the flight were shown.

\section{References}

${ }^{1}$ Rausch, V. L., McClinton, C. R., and Crawford, J. L., "Hyper-X: Flight Validation of Hypersonic Airbreathing Technology," International Society for Air Breathing Engines, ISABE Paper 97-7024, September 1997.

${ }^{2}$ Freeman, D. C., Reubush, D. E., McClinton, C. R., Rausch, V. L., and Crawford, J. L., "The NASA Hyper-X Program," 48th International Astronautical Congress, October 1997.

${ }^{3}$ Harsha, P. T., Keel, L., Castrogiovanni, A., and Sherrill, R. T., "X-43A Vehicle Design and Manufacture," American Institute of Aeronautics and Astronautics, AIAA Paper 2005-3334, May 2005.

${ }^{4}$ Joyce, P. J., Pomroy, J. B., and Grindle, L., "The Hyper-X Launch Vehicle: Challanges and Design Considerations for Hypersonic Flight Testing," American Institute of Aeronautics and Astronautics, AIAA Paper 2005-3333, May 2005.

${ }^{5}$ McClinton, C. R., Rausch, V. L., Nguyen, L. T., and Sitz, J. R., "Preliminary X-43 Flight Test Results," Acta Astronautica, Vol. 57, No. 2-8, 2005, pp. 266-276.

${ }^{6}$ Marshall, L., Corpening, G., and Sherrill, R., "A Chief Engineer's View of NASA's X-43A Scramjet Flight Test," American Institute of Aeronautics and Astronautics, AIAA Paper 2005-3332, May 2005.

${ }^{7}$ Marshall, L., Bahm, C., Corpening, G., and Sherrill, R., "Overview With Results and Lessons Learned of the X-43A Mach 10 Test Flight," American Institute of Aeronautics and Astronautics, AIAA Paper 2005-3336, May 2005.

${ }^{8}$ Karlgaard, C. D., Tartabini, P. V., Blanchard, R. C., Kirsch, M., and Toniolo, M. D., "Hyper-X Post-Flight Trajectory Reconstruction," American Institute of Aeronautics and Astronautics, AIAA Paper 2004-4829, August 2004. Also; Journal of Spacecraft and Rockets, to appear.

${ }^{9}$ Karlgaard, C. D., Tartabini, P. V. and Martin, J. G., "Best-Estimated Trajectory Reconstruction for the X-43A Mach 7 and 10 Flights," JANNAF 40th Combustion Subcommittee, 28th Airbreathing Propulsion Subcommittee, 22nd Propulsion Systems Hazards Subcommittee, and 4th Modeling and Simulation Subcommittee Joint Meeting, Charleston, SC, June 2005.

${ }^{10}$ Karlgaard, C. D., Tartabini, P. V., Martin, J. G., Blanchard, R. C., Kirsch, M., Toniolo, M. D., and Thornblom, M. N., "Statistical Estimation Methods for Hyper-X Trajectory Reconstruction," NASA TM, submitted.

${ }^{11}$ Wagner, W. E., "Re-Entry Filtering, Prediction and Smoothing," Journal of Spacecraft and Rockets, Vol. 3, No. 9, 1966 , pp. 1321-1327. 1970.

${ }^{12}$ Wagner, W. E. and Serold, A. C., "Formulation on Statistical Trajectory Estimation Programs," NASA CR-1482, January

${ }^{13}$ Gelb, A. (Ed.), Applied Optimal Estimation, The M.I.T. Press, Cambridge, MA, 1974.

${ }^{14}$ Fraser, D. C. and Potter, J. E., "The Optimum Linear Smoother as a Combination of of Two Optimum Linear Filters," IEEE Transactions on Automatic Control, Vol. 14, No. 8, 1969, pp. 387-390.

${ }^{15}$ Rogers, R. M., Applied Mathematics in Integrated Navigation Systems, American Institute of Aeronautics and Astronautics, Reston, VA, 2000.

${ }^{16}$ Vallado, D. A., Fundamentals of Astrodynamics and Applications, McGraw-Hill, New York, NY, 1997.

${ }^{17}$ Sofair I., "Improved Method for Calculating Exact Geodetic Latitude and Altitude," Journal of Guidance, Control, and Dynamics, Vol. 20, No. 4, 1997, pp. 824-826.

${ }^{18}$ Sofair, I., "Improved Method for Calculating Exact Geodetic Latitude and Altitude Revisited," Journal of Guidance, Control, and Dynamics, Vol. 23, No. 2, 2000, pp. 369

19 "WGS-84 Implementation Manual," Version 2.4, February 1998.

${ }^{20}$ Phillips, W. F., Hailey, C. E., and Gebert, G. A., "Review of Attitude Representations Used for Aircraft Kinematics," Journal of Aircraft, Vol. 38, No. 4, 2001, pp. 718-737.

${ }^{21}$ Toniolo, M. D., "Flight Data Preprocessing Tool (PREPR) User's Guide," Analytical Mechanics Associates, Inc., AMA Report 04-16, July 2004.

${ }^{22}$ Morelli, E. A., "Estimating Noise Characteristics from Flight Test Data Using Optimal Fourier Smoothing," Journal of Aircraft, Vol. 32, No. 4, 1995, pp. 689-695.

${ }^{23}$ Morelli, E. A., "System Identification Programs for Aircraft (SIDPAC)," American Institute of Aeronautics and Astronautics, AIAA Paper 2002-4702, August 2002.

24 "H-764 System Description," Honeywell, Inc., Report 1096-1339, February 1997.

25 "Hyper-X Flight Management Unit System Performance During Flight Profile Results," Honeywell, Inc., July 1998.

${ }^{26}$ Lin, Y., "X-43A Research Vehicle \#3 Results of FMU Bore Sight Measurements," Hyper-X Report HX-DFRC-0331, Rev. B, September 23, 2004.

${ }^{27}$ Teets, E., "X-43A Flight \#3 Reference Atmosphere," Hyper-X Report HX-DFRC-0421, December 16, 2004.

${ }^{28}$ Marshall, L., "Hyper-X Flight System Performance Requirements," HX-280, Rev. H., June 8, 2004.

${ }^{29}$ Tartabini, P. V., "Mach 10 Stage Separation Design and Analysis Report," HX-DFRC-0408, October 27, 2004. 
${ }^{30}$ Blocker, W. D. and Reubush, D. E., "X-43A Stage Separation System - A Flight Data Evaluation," American Institute of Aeronautics and Astronautics, AIAA Paper 2005-3335, May 2005.

${ }^{31}$ Ferlemann, P. G., "Comparison of Hyper-X Mach 10 Scramjet Preflight Predictions and Flight Data," American Institute of Aeronautics and Astronautics, AIAA Paper 2005-3352, May 2005.

${ }^{32}$ Baumann, E., Bahm, C., Beck, R., and Strovers, B., "X-43A Mach 10 Mission Guidance and Control Updates, Rationale, and Flight Test Results," JANNAF 40th Combustion Subcommittee, 28th Airbreathing Propulsion Subcommittee, 22nd Propulsion Systems Hazards Subcommittee, and 4th Modeling and Simulation Subcommittee Joint Meeting, Charleston, SC, June 2005

${ }^{33}$ Morelli, E. A., Derry, S. D., and Smith, M., "Aerodynamic Parameter Estimation for the X-43A from Flight Data," American Institute of Aeronautics and Astronautics, AIAA Paper 2005-5921, August 2005. 